\title{
LARINGECTOMIA SUPRACRICÓIDE (CHEP) PARA CÂNCER GLÓTICO
}

\section{SUPRACRICOID LARYNGECTOMY (CHEP) FOR GLOTTIC CANCER}

\author{
Roberto R. M.Araújo Lima, TCBC-RJ ${ }^{1}$ \\ Emilson de Queiroz Freitas, TCBC-RJ'2 \\ Jacob Kligerman, TCBC-R. ${ }^{3}$ \\ Geraldo Matos de Sá, TCBC-RJ ${ }^{4}$ \\ Izabella Costa Santos ${ }^{2}$ \\ Terence Farias ${ }^{2}$
}

\begin{abstract}
RESUMO: Objetivos: Com a intenção de se avaliar os resultados funcionais e terapêuticos da laringectomia supracricóide com crico-hióide-epiglote-pexia (CHEP) foram estudados 50 casos de pacientes com carcinoma epidermóide da glote classificados como T2/T3 submetidos a esta técnica em nossa instituição. Métodos: Foi realizada uma análise retrospectiva entre 1996 e 1999. Classificamos 18 pacientes como T2N0M0 e 32 pacientes como T3N0M0. Quarenta e um pacientes foram submetidos a esvaziamento cervical seletivo lateral bilateralmente, quatro foram submetidos ao mesmo esvaziamento unilateralmente, e cinco não foram esvaziados. Analisamos as complicações e a sobrevida livre de doença pelo método de Kaplan-Meyer. Resultados: Dez pacientes tiveram complicações pós-operatórias, dois foram tratados com complementação da laringectomia. Os 48 pacientes restantes mantiveram a via aérea normal, deglutição e a voz. Três pacientes no grupo submetido a esvaziamento cervical apresentaram linfonodo metastático. Quatro pacientes tiveram recidiva da doença, três com recidiva local, sendo dois tratados com laringectomia total e estão vivos e sem doença, o outro com doença avançada alcançou o óbito pela doença. $\mathrm{O}$ paciente que teve recidiva no pescoço foi tratado com esvaziamento cervical mais radioterapia e morreu com doença. Dois pacientes tiveram um segundo tumor primário em orofaringe, sendo um tratado com radioterapia paliativa e morreu com doença e o outro tratado com cirurgia está vivo e sem doença. A sobrevida livre de doença em três anos foi de $88 \%$ para pacientes T2 e $72 \%$ para pacientes T3. Conclusões: Esta técnica é útil no tratamento de casos selecionados de carcinoma epidermóide da glote T2/T3 sempre se considerando a extensão da doença. A incidência de complicações necessitando laringectomia total de resgate não compromete a funcionalidade desta técnica. A sobrevivência é comparável aos pacientes submetidos a laringectomia total e laringectomia "near-total".
\end{abstract}

Descritores: Laringectomia, Laringectomia parcial, Câncer da laringe, Laringe.

\section{INTRODUÇÃO}

O objetivo do tratamento do câncer da laringe é a cura e preservação da voz quando possível. Historicamente o tratamento cirúrgico para os pacientes com lesões avançadas da laringe estádio três tem sido a laringectomia total. Recentemente têm sido desenvolvidas novas técnicas cirúrgicas que obtêm a radicalidade necessária, preservando a voz. Nos Estados Unidos, Bruce Pearson ${ }^{1}$ idealizou a laringectomia "near-total", que promovia a erradicação

1.Titular da Seção de Cirurgia de Cabeça e Pescoço Hospital do Câncer - INCA. Professor da Disciplina de Cirurgia de Cabeça e Pescoço da Universidade Gama Filho. Doutorando em Clínica Cirúrgica da USP.

2. Titular da Seção de Cirurgia de Cabeça e Pescoço Hospital do Câncer - INCA.

3. Diretor Geral do Instituto Nacional de Câncer. Titular da Seção de Cirurgia de Cabeça e Pescoço do Hospital do Câncer - INCA.

4. Titular da Seção de Cirurgia de Cabeça e Pescoço do Hospital do Câncer - INCA. Professor Titular da Disciplina de Cancerologia da Universidade Souza Marques. Professor Titular da Disciplina de Cirurgia de Cabeça e Pescoço da PUC.RJ.

Recebido em 14/9/2000

Aceito para publicação em 6/3/2001

Trabalho realizado na Seção de Cirurgia de Cabeça e Pescoço, Hospital do Câncer / INCA, Rio de Janeiro, BRASIL. 
da doença com a manutenção da voz em casos de tumores malignos da laringe, principalmente o carcinoma epidermóide, que acometia apenas um lado do órgão com fixação do mesmo. Após 1990, iniciamos a utilização desta operação em nosso serviço ${ }^{2}$. Esta técnica mantém um shunt estreito comunicando a traquéia e a faringe, possibilitando a emissão de sons com a vibração da aritenóide remanescente. Porém este shunt é muito estreito para permitir a passagem do ar necessário à respiração, obrigando a manutenção de traqueostomia definitiva.

Certas lesões glóticas da laringe classificadas como T2 ou T3 necessitando laringectomia total para ressecção do tumor com segurança oncológica tinham como opção cirúrgica a laringectomia "near-total". Entretanto, consideramos que em lesões T2 ou T3 glóticas a operação de Pearson é muito extensa, sobretudo para casos de doença unilateral com fixação da corda vocal com aritenóide móvel ou lesões glóticas com invasão de algumas regiões da supraglote.

Após 1996, com o conhecimento dos trabalhos de autores franceses ${ }^{3,4}$, iniciamos em nosso serviço o emprego da laringectomia supracricóide com crico-hióide-epiglote-pexia (CHEP). Consideramos que está técnica que vinha sendo utilizada principalmente em lesões $\mathrm{T} 2$ poderia ter sua indicação ampliada em casos selecionados de lesões T3 glóticas 5 .

Esta abordagem permite a ressecção de tumores glóticos, associada à exérese do espaço paraglótico, quando se incluem ambas as cordas vocais e toda a cartilagem tireóide na peça operatória (Figura 1). Após a ressecção, a reconstrução da via aérea é feita com a elevação e fixação da cartilagem cricóide na epiglote e no osso hióide (Figura 2). Sendo, portanto, possível retirar a traqueostomia e manter voz, rouca, porém perfeitamente inteligível.

Em pacientes que apresentam lesões glóticas mais extensas, que alcançam a apófise vocal, é possível a ressecção de uma das aritenóides para segurança oncológica.

Com a intenção de avaliar a eficácia desta técnica no tratamento de casos selecionados de lesões glóticas, estudamos 50 casos de pacientes com carcinoma epidermóide da glote tratados em nossa instituição com esta operação.

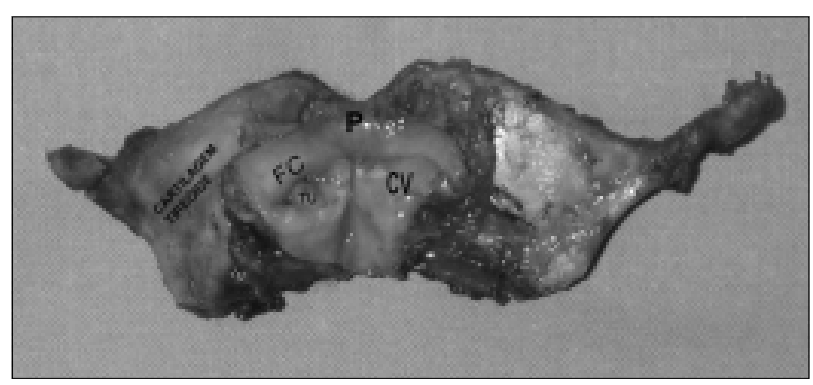

Figura 1 - Peça operatória de CHEP. ( $P=$ Pecíolo da epiglote, $C V=$ Corda vocal, $F C=$ Falsa corda, $T U=$ Tumor $)$

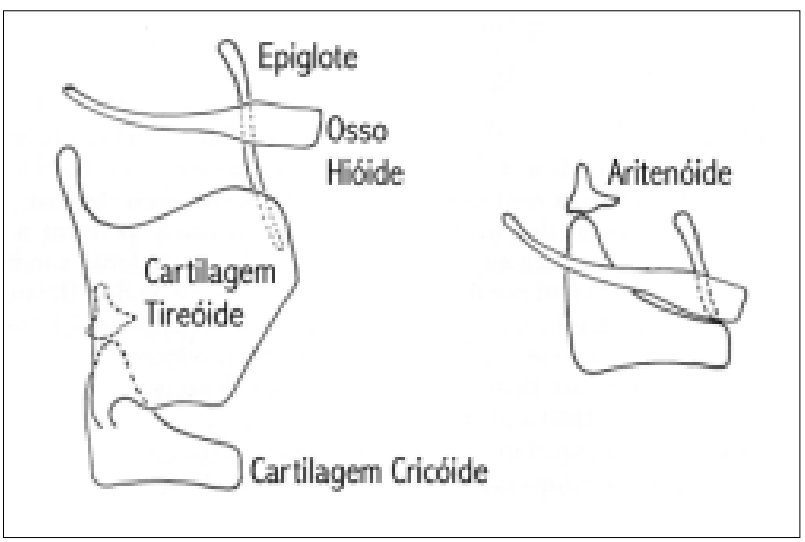

Figura 2 - Reconstrução da via aérea com crico-hióide-epiglotepexia.

\section{MÉTODO}

Foi realizada análise retrospectiva de 50 pacientes, com carcinoma epidermóide T2/T3 da glote, tratados em nossa instituição entre 1996 e 1999.

A idade média foi de 57 anos (intervalo de 41 a 79). Quarenta e seis pacientes eram do sexo masculino e quatro do sexo feminino. Todos os pacientes eram tabagistas, sendo 17 deles também etilistas. Quarenta e três pacientes apresentavam lesões glóticas e sete pacientes apresentavam lesões transglóticas. Todos foram avaliados segundo a classificação TNM da UICC de 1998, sendo 18 pacientes classificados como T2NOM0 e 32 pacientes como T3NOM0 (Tabela 1). Todos os pacientes foram avaliados por videolaringoscopia, laringoscopia direta e tomografia computadorizada da laringe.

Tabela 1

Local e estágio dos tumores tratados com CHEP

\begin{tabular}{lcc}
\hline & T2NOMO & T3NOMO \\
\hline Glótico & 18 & 25 \\
Transglótico & 0 & 7 \\
\hline
\end{tabular}

Seis pacientes tiveram o tratamento cirúrgico complementado por radioterapia em casos de margens cirúrgicas próximas ou invasão sem extravasamento da cartilagem tireóide. Nossas indicações de CHEP incluíram os casos de lesões T3 glóticas unilaterais e/ou anteriores com fixação de corda vocal, porém com aritenóide móvel e sem invasão de infraglote além da região correspondente à borda inferior da corda vocal. Os 18 pacientes com lesões T2 apresentavam invasão do ventrículo de Morgani ou redução da mobilidade da corda vocal. Quarenta e um pacientes foram submetidos a esvaziamento cervical lateral bilateralmente ${ }^{6}$, quatro pacientes foram submetidos ao mesmo 
esvaziamento unilateralmente e cinco pacientes não foram esvaziados. A sobrevida livre de doença foi analisada pelo método de Kaplan-Meier.

\section{RESULTADOS}

Obtivemos sucesso empregando esta técnica em 48 pacientes. Dois pacientes apresentaram quadro de broncoaspiração grave. Um deles teve redução da mobilidade da aritenóide remanescente provocando quadro grave de broncoaspiração com pneumonias repetidas e foi submetido à complementação de laringectomia. Outro paciente apresentou ruptura da pexia, com aspiração brônquica repetida, sendo submetido à complementação de laringectomia. Cinco pacientes apresentaram redundância de mucosa do lado da aritenóide ressecada causando obstrução respiratória leve e moderada sendo tratados com ressecção da mucosa com laser de $\mathrm{CO}_{2}$. Um paciente apresentou fístula faringocutânea, sendo tratado com medidas conservadoras. Dois pacientes apresentaram estenose da neoglote, sendo tratados com alargamento por ressecção a laser (Tabela 2).

Tabela 2

Complicações pós-operatórias

\begin{tabular}{lcc}
\hline & Freqüência & $\%$ \\
\hline $\begin{array}{l}\text { Broncoaspiração grave } \\
\text { (ruptura da pexia e paralisia } \\
\text { da aritenóide) }\end{array}$ & 2 & 4 \\
Estenose & 2 & 4 \\
Fístula faringocutânea & 1 & 2 \\
Mucosa redundante & 5 & 10 \\
\hline
\end{tabular}

Quarenta e oito pacientes tiveram a traqueostomia decanulada com sucesso. O tempo médio de decanulação da traqueostomia foi de 33 dias (intervalo de oito a 120 dias), o paciente com mucosa redundante necessitou de traqueostomia por 120 dias até a decisão de usar o laser para ressecar a mucosa redundante. $\mathrm{O}$ tempo médio de sonda nasoenteral foi de 38 dias (intervalo de sete a 120 dias).

Três pacientes submetidos a esvaziamento cervical apresentaram metástase cervical oculta.

Quatro pacientes apresentaram recidiva da doença. Um paciente classificado como T2 do grupo de pacientes não esvaziados apresentou recidiva cervical em nível 3 e foi tratado com esvaziamento cervical radical modificado complementado por radioterapia evoluindo para êxito letal após três meses (Tabela 3 ).

Três pacientes, com lesões T3, recidivaram localmente sendo dois tratados com complementação da laringectomia e estão vivos sem doença. O outro paciente apresentava doença avançada e faleceu sem tratamento.

Dois pacientes apresentaram um segundo tumor primário em orofaringe sendo um tratado com radiote-
Tabela 3

Incidência de recidiva cervical e metástase cervical oculta

\begin{tabular}{l|c|c}
\hline & $\begin{array}{c}\text { Recidivas } \\
\text { cervicais }\end{array}$ & $\begin{array}{c}\text { Metástase } \\
\text { oculta }\end{array}$ \\
\hline Esvaziamento cervical bilateral & $0 / 19$ & $3 / 41$ \\
Esvaziamento cervical unilateral & $0 / 3$ & $0 / 4$ \\
Sem esvaziamento cervical & $1 / 5$ & - \\
\hline
\end{tabular}

rapia paliativa alcançando o óbito pela doença após seis meses e o outro foi tratado cirurgicamente e está vivo sem doença.

A sobrevida livre de doença em três anos foi de $88 \%$ e $72 \%$ para os pacientes classificados como T2 e T3, respectivamente.

\section{DISCUSSÃO}

A maioria dos pacientes com câncer da laringe atendidos em nossa instituição apresentou lesões avançadas, principalmente lesões T3, foi tratada com laringectomia total $^{7}$ em campo alargado ou laringectomia "near-total" 2 .

As opções de reabilitação vocal para os pacientes submetidos à laringectomia total são a colocação de prótese vocal, a voz esofagiana ou o uso do dispositivo vibratório conhecido como voz eletrônica. Muito destes pacientes moram distante do hospital e apresentam baixas condições socioeconômicas o que dificulta a reabilitação com próteses vocais que necessitam de freqüentes $\operatorname{trocas}^{8}$ e visitas ao hospital, além disso, os bons candidatos para o uso de tais próteses necessitam de boa habilidade manual e razoável compreensão da anatomia ${ }^{9}$. Da mesma maneira o sucesso do treinamento com a voz esofagiana está diretamente relacionado a uma capacitação difícil para o paciente e a algumas características próprias como a capacidade de retenção de ar no estômago. Assim, não são todos os pacientes que conseguem se comunicar por este meio. A voz eletrônica é dependente de um dispositivo eletrônico mantido por baterias, o que acarreta um custo de funcionamento elevado para alguns pacientes, além de também necessitar de treinamento adequado e de capacidade de se adaptar ao dispositivo.

Portanto, estes métodos de reabilitação são dependentes ou de dispositivos externos ou da capacidade individual de cada paciente.

As laringectomias conservadoras, tanto "near-total" como a supracricóide, permitem ao paciente a reabilitação vocal sem o uso de qualquer dispositivo e sem muito treinamento.

Além disso, tratar casos selecionados de lesões T3 com aritenóide móvel ou lesões T2 com invasão de supraglote ou redução da mobilidade da corda vocal com a laringectomia "near-total" nos parecia uma ressecção muito extensa. Além do mais, estudos demonstraram que um dos fatores de maior impacto na sobrevida era a manutenção da traqueostomia ${ }^{10}$. 


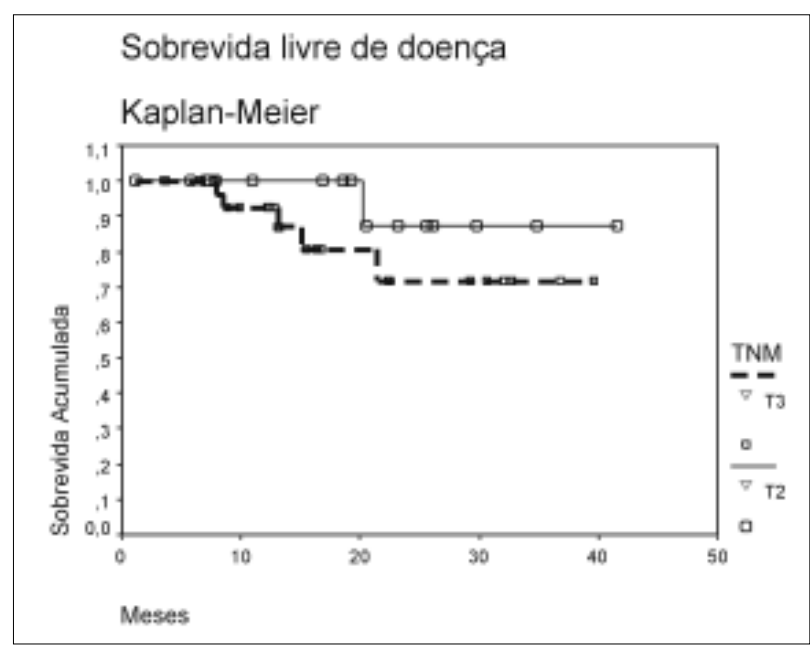

Figura 3 - Sobrevida livre de doença em três anos.

Outra opção terapêutica para lesões da glote moderadamente avançadas é a radioterapia exclusiva, ou associada à quimioterapia deixando-se o tratamento cirúrgico para resgate em casos de recidiva ou doença residual ${ }^{11,12}$. Esta opção terapêutica consegue preservar a laringe em cerca de $65 \%$ dos $\operatorname{casos}^{12}$. Contudo este é um tratamento de alto custo e que necessita de pacientes em boas condições físicas não muito comuns em nosso meio. Além disso, não obtivemos muito sucesso com este tratamento devido principalmente às precárias condições físicas de nossos pacientes provocando a descontinuidade do tratamento por não adequação às drogas utilizadas.

Consideramos também que a grande maioria dos pacientes submetidos a resgate cirúrgico por falha no tratamento quimiorradioterápico é beneficiada pela laringectomia total.

A partir de 1996 e após o conhecimento de trabalhos de Piquet $^{3}$ e Laccourreye ${ }^{4}$, decidimos tratar casos selecionados de lesões glóticas T2/T3 com a CHEP. Nos baseamos no conhecimento de que pacientes submetidos a CHEP mantinham a voz, a via aérea fisiológica, assim como a deglutição por via oral, além da segurança oncológica deste procedimento ter sido motivo de várias publicações em periódicos especializados $3,4,13,14-18$.

Diferentemente de outros estudos ${ }^{5}, 64 \%$ de nossos pacientes apresentavam lesões mais avançadas no momento do diagnóstico.

Apesar de dois de nossos pacientes terem tido complicações que necessitaram complementação da laringectomia, 48 pacientes (96\%) mantiveram satisfatoriamente a via aérea natural, assim como a voz e a deglutição, resulta- dos similares à outra casuística ${ }^{5}$. O tempo médio até a retirada da traqueostomia e cateter nasoenteral foi maior que de outros estudos ${ }^{19-22}$, este fato pode ser explicado pela baixa condição socioeconômica dos nossos pacientes.

Quatro casos de ruptura de pexia relatados por Laccourreye $^{16}$ foram tratados com complementação de laringectomia em um caso e revisão da pexia em três, com sucesso. Fatores como cartilagem cricóide não ossificada e ressecção parcial da referida estrutura foram associados a esta complicação. Tivemos um caso de ruptura, porém o paciente não concordou com a revisão, sendo submetido à complementação da laringectomia.

A maioria de nossas complicações ocorreu devido ao excesso de mucosa pendendo para a neoglote como consequiência de erro técnico motivado pela ansiedade de corrigir o defeito local.

Todos pacientes classificados como T3 foram submetidos a esvaziamento cervical seletivo lateral, dois apresentaram metástase cervical oculta (Tabela 3). Um, classificado como T2, apresentou metástase cervical oculta e um dos T2 não submetidos a esvaziamento cervical apresentou recidiva no pescoço o que pode ser explicado pela invasão da supraglote.

Vários estudos ${ }^{23-25}$ têm demonstrado a alta incidência de metástase cervical oculta ou detectada no momento do diagnóstico em tumores glóticos e supraglóticos, sendo razoável assumir que as metástases ocultas de carcinoma epidermóide aparecerão se não forem tratadas. Além do mais, estudos ${ }^{7,26}$ têm demonstrado um impacto adverso no prognóstico dos pacientes em que a metástase cervical foi tratada após o seu aparecimento, o que freqüentemente ocorria em estágios mais avançados. Em nosso meio onde o seguimento dos pacientes operados é feito com alguma dificuldade, este fato se agrava, e quando os pacientes retornam para o controle já apresentam metástase cervical muito avançada, e, com freqüência, inoperável.

A sobrevida livre de doença em nossa série foi similar a outros estudos $4,5,13,19,22$. Entretanto, devemos levar em consideração que os pacientes com indicação de tratamento cirúrgico com a laringectomia supracricóide apresentam lesões limitadas, mesmo os mais avançados (T3).

Esta técnica pode ser indicada tanto em pacientes classificados como T2 ou T3, sempre se considerando a extensão da doença. A incidência de complicações que necessitaram de tratamento com complementação de laringectomia não compromete a funcionalidade da técnica.

A sobrevida livre de doença em três anos é comparável à sobrevida de lesões glóticas com a mesma extensão e submetidas a laringectomia total ou laringectomia "near-total".

\footnotetext{
ABSTRACT

Background: Our objective was to assess whether Supracricoid Laryngectomy with CricoHiodoEpiglottoPexy (CHEP) could successfully achive cure preserving voice in the treatment of glottic laryngeal cancer. Methods: A retrospective analysis has been carried out between 1996 through 1999. We classified 18 patients as T2NOMO and 32 patients as T3NOMO. Fourty one patients underwent selective bilateral lateral neck dissection, four had unilateral neck dissection and five patients had the neck undissected. Survival was analyzed under the KaplanMeyer method. Results: Ten patients had postoperative complications, two treated with total laryngectomy. The remained 48 patients maintained normal airway, swallowing and speech. Three patients in the neck dissection
} 
group presented occult neck metastasis. Four patients had recurrences, three of them were local. Two patients treated with total laryngectomy are alive without disease, and another had advanced recurrence and died. One patient had neck recurrence and was treated with radical neck dissection plus radiotherapy and died from the disease. Two patients presented a second tumor in the oropharynx. One of them was treated with palliative radiotherapy and died and the other had surgical resection and is alive without disease. Three years disease free survival was $88 \%$ for T2 and 72\% for T3. Conclusions: This technique is useful in the treatment of selected cases of T3/T2 glottic cancer, regarding the extension of the disease. The incidence of complications required completion laryngectomy which did not compromise the functionality of this technique. Survival is comparable to patients submitted to total laryngectomy and near-total laryngectomy, regarding the extension of the lesion.

Keywords: Larynx, Cancer, Supracricoid, Laryngectomy, Squamous cell carcinoma, Partial laryngectomy.

\section{REFERÊNCIAS}

1. Pearson, BW. "The Theory and Technique of Near-otal Laryngectomy”. In. Bailey, BJ and Biller, H. J. (eds) Surgery of the Larynx. Philadelphia: W.B. Saunders Company, 1985, pp 333-346.

2. Lima RA, Freitas EQ, Kligerman J, et al. Near-Total Laryngectomy for Treatment of Advanced Laryngeal Cancer. Am J Surg 1997; 174:490-491.

3. Piquet $\mathbf{J}$ and Chevalier D. Subtotal Laryngectomy With Cricohioidoepiglottopexy for the Treatment of Extended Glottic Carcinomas. Am J Surg 1991; 162:357-361.

4. Laccourreye H, Laccourreye O, Weinstein G, et al. Supracricoid Laryngectomy With Cricohyoidoepiglottopexy: A Partial Laryngeal Procedure For Glottic Carcinoma. Ann Otol Rhinol Laryngol 1990; 99:421-426.

5. Lefebvre J and Chevalier D. Supracricoid Partial Laryngectomy. Advances in Otolaryngology-Head and Neck Surgery $1998 ; 12: 1-15$.

6. Robbins KT, Medina JE, Wolfe GT, et al. Standardizing Neck Dissection Terminology: Official Report of the Academy's Committee for Head and Neck Surgery and Oncology. Arch Otolaryngol Head Neck Surg 1991; 117:601605.

7. Kligerman J, Olivatto LO, Lima RA, et al. Elective Neck Dissection in the Treatment of T3/T4 N0 Squamous Cell Carcinoma of the Larynx. Am J Surg 1995; 170: 436-439.

8. Levine PA, Debo RF, Reibel JF. Pearson Near-Total Laryngectomy: A Reproducible Speaking Shunt. Head \& Neck 1994;16:323-325.

9. Andrews JC, Mickel RA, Hanson DG, et al. Major Complications Following Tracheoesophageal Puncture For Voice Rehabilitation. Laryngoscope 1987; 97:562-567.

10. DeSanto LW, Olsen KD, Perry WC, et al. Quality of Life After Surgical Treatment of Cancer of the Larynx. Ann Otol Rhinol Laryngol 1995; 104:763-769.

11. Sandberg N, Mercke C,Turesson I. Glottic Laryngeal Carcinoma With Fixed Vocal Cord Treated With FullDose Radiation, Total Laryngectomy or Combined Treatment. Acta Oncologica 1990; 29:509-511.

12. Wolf GT, Hong WK, Fisher SG, et al. Induction Chemotherapy Plus Radiation Compared With Surgery Plus Radiation in Patients With Advanced Laryngeal Cancer. N Engl J Med 1991; 324:1685-1690.

13. Chevalier D and Piquet J. Subtotal Laryngectomy With Cricohyoidopexy for Supraglottic Carcinoma: Review of 61 Cases. Am J Surg 1994; 168:472-473.

14. Laccourreye O, Salzer SJ, Brasnu D, et al. Glottic Carcinoma With a Fixed True Vocal Cord: Outcomes After Neoadjuvant Chemotherapy and Supracricoid Partial
Laryngectomy With Cricohyoidoepiglottopexy. Otolaryngol Head Neck Surg 1996; 114:400-406.

15. Laccourreye O, Muscatello L, Laccourreye L, et al. Supracricoid partial laryngectomy with cricohyoidoepiglottopexy for "early" glottic carcinoma classified as T1T2N0 invading the anterior commissure. Am J Otolaryngol 1997; 18:385-390.

16. Laccourreye O, Brasnu D, Laccourreye L, et al. Ruptured pexis after supracricoid partial laryngectomy. Ann Otol Rhinol Laryngol 1997; 106:159-162.

17. Laccourreye O, Laccourreye L, Muscatello L, et al. Local failure after supracricoid partial laryngectomy: symptoms, management, and outcome. Laryngoscope 1998; 108:339-344.

18. Laccourreye O, Brasnu D, Périé S, et al. Supracricoid partial laryngectomies in the elderly: mortality, complications, and functional outcome. Laryngoscope 1998; 108:237-242.

19. de Vincentiis M, Minni A, Gallo A, et al. Supracricoid Partial Laryngectomies: Oncologic and Functional Results. Head \& Neck 1998; 20:504-509.

20. Naudo P, Laccourreye O, Weinstein G, et al. Complications and functional outcome after supracricoid partial laryngectomy with cricohyoidoepiglottopexy. Otolaryngol Head Neck Surg 1998; 118:124-129.

21. Rassekh CH, Driscoll BP, Seikaly H, et al. Preservation of the superior laryngeal nerve in supraglottic and supracricoid partial laryngectomy. Laryngoscope 1998; 108:445-447.

22. Weinstein GS and Laccourreye O. Supracricoid Laryngectomy With Cricohyoidepiglottopexy. Otolaryngol Head Neck Surg 1994; 111:684-685.

23. Johnson JT, Myers EN, Hao S, et al. Outcome of Open Surgical Therapy for Glottic Carcinoma. Ann Otol Rhinol Laryngol 1993; 102:752-755.

24. Pitman KT. Rationale for elective neck dissection. Am J Otolaryngol 2000; 21:31-37.

25. Myers EN and Fagan JJ. Management of the Neck in Cancer of the Larynx. Ann Otol Rhinol Laryngol 1999; 108:828-832.

26. Silver CE and Moisa II. Elective Treatment of the Neck in Cancer of the Oral Tongue. Seminars in Surgical Oncology 1991; 7:14-19.

Endereço do autor:

Dr. Roberto Araújo Lima

Rua Real Grandeza, 139/606.

22281-030 - Rio de Janeiro-RJ

Fax: (21) 2266-3165

E-mail:rlima@inca.org.br 